\title{
JEM-EUSO observational capabilities for different UHE primaries
}

\author{
Alejandro Guzmán*, Andrea Santangelo, Elias Iwotschkin, Thomas Mernik, Jörg \\ Bayer, Kenji Shinozaki \\ Insitute für Astronomie und Astrophysik, Univeristät Tübingen, Germany \\ E-mail: guzman@astro.uni-tuebingen.de
}

\section{Francesco Fenu, Mario Bertaina}

Dipartimento di Fisica, Universita' di Torino, Italy

\section{Gustavo Medina-Tanco}

Insituto de Ciencias Nucleares, UNAM, México

\section{Angela V. Olinto}

Kavil Insitute for Cosmological Physics, University of Chicago, USA

\section{Lawrence Wiencke}

Department of Physics, Colorado School of Mines, USA

\section{for the JEM-EUSO Collaboration}

Cosmic rays with energies exceeding $10^{18} \mathrm{eV}$, usually defined as Ultra High Energy Cosmic Rays (UHECRs), allow the possibility to study physics at energies well beyond man made accelerators. State of the art UHECR detectors have reached unprecedented exposures and have pioneered the field of Extreme Energy Cosmic Rays (EECR), cosmic rays with energies exceeding $5 \times 10^{19} \mathrm{eV}$. The EECR flux is extremely small, of the order of 1 particle per square kilometer per century. The next generation of UHECR and EECR detectors are expected to increase the exposure by at least one order of magnitude. The JEM-EUSO mission, currently designed to be hosted onboard the JEM module of the ISS, consists of a ultra wide field of view UV-telescope orbiting the earth at an altitude of about $400 \mathrm{~km}$. JEM-EUSO will look for fluorescent UV tracks produced by Extensive Air Showers (EAS) on the night side of the earth. According to the most recent studies, the JEMEUSO mission, can be transported onto the ISS by using the SpaceX's Dragon spacecraft. In this work we present preliminary studies on the angular and energy reconstruction performances for different types of primaries (protons, iron nuclei and gamma rays). We compare our results with previously published results for the JEM-EUSO mission in a different configuration, and find a slight improvement.

The 34th International Cosmic Ray Conference,

30 July- 6 August, 2015

The Hague, The Netherlands

\footnotetext{
* Speaker.
} 


\section{Introduction}

The planned Extreme Universe Space Observatory on the Japanese Experiment Module (JEMEUSO), is a downward looking telescope designed to detect the ultraviolet photons produced by extended air showers in the Earth's atmosphere. JEM-EUSO is equipped with three Fresnel lenses that focus the UV photons onto the focal surface (FS) detector. This FS detector is made of 137 individual photo-detector modules (PDMs), and each PDM is formed by 36 multi-anode photomultiplier tubes (MAPMT). Each MAPMT has $8 \times 8=64$ pixels. An operation height of $400 \mathrm{~km}$, combined with JEM-EUSO's super wide angle field of view (FoV), will provide unprecedented exposure to Extreme Energy Cosmic Rays (EECR). The expected value of the viewing angle is $\sim \pm 30^{\circ}$. In combination with the aforementioned altitude, this translates into an instantaneous monitored surface $\sim 150000$ square kilometers. The telescope will also be equipped with an atmospheric monitoring system consisting of a LIDAR system and IR-camera to record the state of the atmosphere including the altitude of clouds inside the field of view. A detailed introduction and of the expected exposure can be found on [1].

\section{The JEM-EUSO mission accommodated in the SpaceX Dragon Spacecraft}

The SpaceX Dragon System (shown in Fig. 1) is capable of delivering cargo to the International Space Station (ISS), and began such operations in 2012[5]. The possibility of using the SpaceX Dragon System to transport the JEM-EUSO detector to the ISS, is currently being explored by the JEM-EUSO collaboration. The Dragon Capsule is launched into orbit on SpaceX's Falcon 9, where it rendezvouses with the ISS. After a successful docking, the unpressurized payload (in our case the JEM-EUSO instrument) is removed by the Robotic Manipulator Arm and docked to the payload attachment point on the JEM module of the ISS [6]. Another possibility previously explored by the JEM-EUSO collaboration, was to accommodate the telescope in the operated HTV-II by JAXA [1] [7].

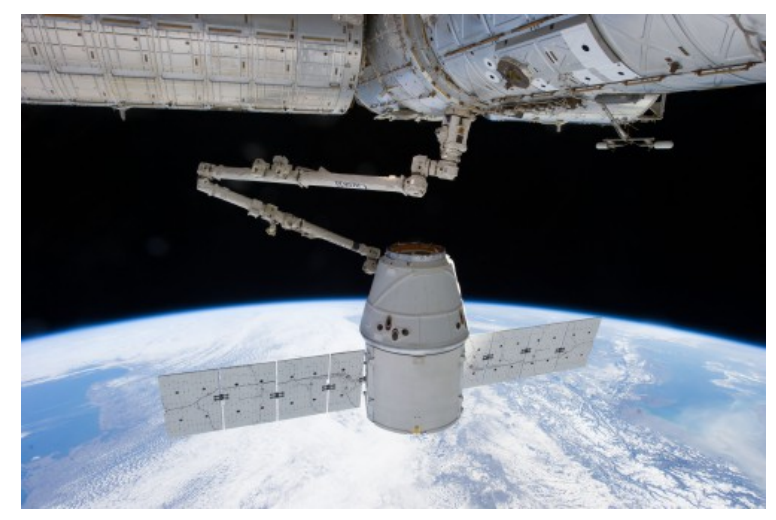

Figure 1: The SpaceX's Dragon berthing on the ISS. The unpressurized cargo compartment is the cylindrical structure from where the solar panels are deploying. Image reproduced from [5]

Although changing the delivery spacecraft does not affect the principle of operation, the specifics of the detector have to be fine-tuned to match the payload constrains of the spacecraft. Particularly the shape of and consequently the optics of the telescope differ from one option to the 
other. This modification is illustrated in Fig. 2. We can see that the dragon option offers an almost circular focal surface layout, whilst conserving the sensitive area.

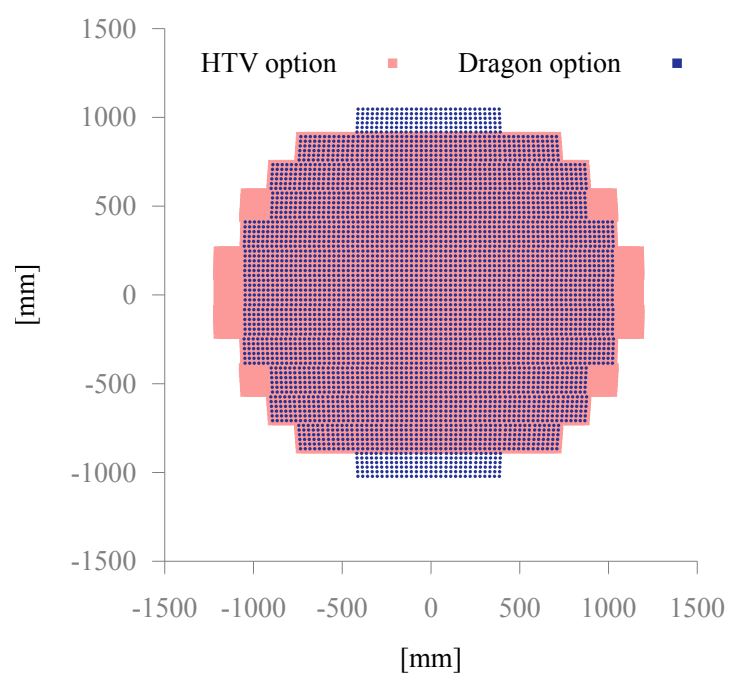

Figure 2: A diagram of the position of the MAPMTs of the Dragon configuration (blue) superimposed on the same diagram but for the HTV configuration (red).

\section{Shower simultations}

To assess the expected behavior of the new configuration of the instrument we carried out simulations using the EUSO Simulations and Analysis Framework ESAF [9]. This software provides an end-to-end simulation of the relevant processes: the EAS development, the UV photon production, the radiative transport of the photons to the telescope, the passage of the UV photons through the optics of the detector, and finally the detector's electronics including its trigger algorithms. Also within the ESAF framework, a set of reconstruction tools has been implemented to calculate the arrival direction, energy and $X_{\max }$, starting from simulated events [17][8] [16].

In previous works aimed to simulate the JEM-EUSO mission, only parametrized showersimulations have been used [1]. Hence the shower-to-shower fluctuations have not been completely taken into account. In this work we used the hybrid EAS-simulator in CONEX[10]. ConEXis a one dimensional simulation software that combines Monte-Carlo (MC) simulations and shower cascade equations to calculate the EAS's main characteristics, i.e number of charged particles, energy deposition profile, number of muons, etc. This approach provides the advantage of reducing the use of computer resources when compared with full MC simulations. The hadronic interactions model we used in this work is the LHC-updated EPOS-LHC [11].

Another advantage of using the CONEX simulation program is that we can study the behavior of the detector in the presence of different primaries. For the present work we present three case scenarios: protons, irons and photons. 

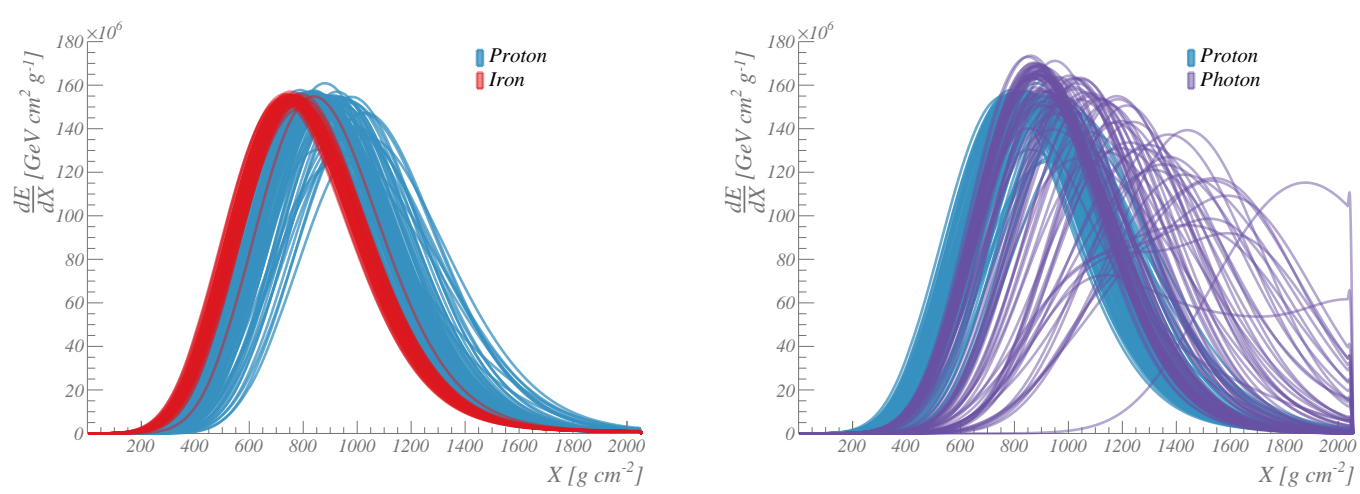

Figure 3: Left: a comparison of the longitudinal profiles of EAS inititated by protons and iron nuclei. For both primaries we show a subsample of 100 showers with an energy of $10^{20} \mathrm{eV}$ and $\Theta=60^{\circ}$. Right : a similar comparison between the longitudinal profiles of EAS initiated by protons and photons $(\gamma)$ injected along the trajectory of the ISS.The abrupt end of the deeply penetrating photon EAS at $\sim 2000 \mathrm{~g} \mathrm{~cm}^{-2}$ marks the shower front reaching the ground.

\subsection{Hadronic primaries}

Though the chemical composition of UHECR and EECR may be in agreement within experimental uncertainties, the issue is still not completely settled by current experiments [14]. Therefore it is illustrative to show the behavior of the future JEM-EUSO mission with two representative hadronic primaries, protons and iron nuclei. For each primary, we have simulated events with energies of $5 \times 10^{19}$ and $10^{20} \mathrm{eV}$. These events have zenith angles of $\Theta=30^{\circ}, 45^{\circ}, 60^{\circ}$ and $75^{\circ}$. For each angle and energy combinations we simulated 1000 events. An example of the longitudinal profiles for protons and iron nuclei is shown in at the left panel of Fig. 3 .

\subsection{Photon primaries}

A EUSO like mission may improve the limits on the photon fraction of UHECR [2]. We simulated a similar sample with $E=10^{20}$ and the same zenith angles, but this time with photons as primaries. Due to possible interactions with the geomagnetic field, special care has to be taken when performing the photon simulations. The pair production process depends strongly on the magnetic component transverse to the photon's direction of motion, and therefore the event simulation is sensitive to the value and direction of the local geomagnetic field [12]. We accounted for this effect in our simulations by sampling the longitude and latitude position of the ISS along its orbit. An example of the simulated longitudinal profiles is shown in the right side of Fig. 3.

\section{Angular reconstruction}

After running the CONEX-simulated shower through ESAF, we proceeded to reconstruct the simulated showers. We used a similar approach as the one presented in [8]. However this time we added an update to the pattern recognition algorithm PWISE [8]. The newer version performs a second iteration over the pixels activated, adding more pixels for the direction reconstruction module. 
We characterize the error in the reconstructed arrival direction as the angle between the simulated arrival direction vector $\hat{\Omega}_{\text {Simu }}$ and its reconstructed counterpart $\hat{\Omega}_{\text {Reco }}$. We define

$$
\gamma=\operatorname{acos}\left(\hat{\Omega}_{\text {True }} \cdot \hat{\Omega}_{\text {Reco }}\right)
$$

as the error in the reconstruction. To compare our results with similar studies previously published [15] [8] we define the angular resolution as the value where the cumulative distribution of the reconstruction's error reaches $68 \%$. We shall refer to this value as $\gamma_{68}$.

\subsection{Angular reconstruction of hadronic primaries}
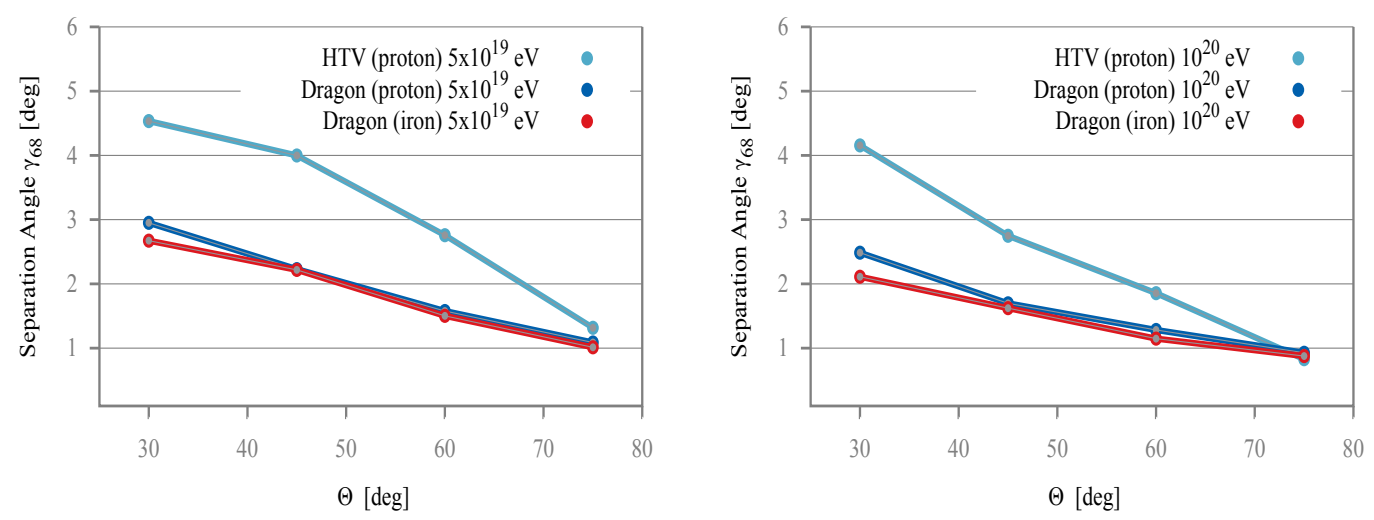

Figure 4: The angular resolution as a function of the zenith angle of the Dragon-configuration of the JEMEUSO mission for proton(dark blue) and iron (red) primaries with an energy of $5 \times 10^{19} \mathrm{eV}$ (left), and $10^{20} \mathrm{eV}$ (right). Previous results with the HTV-configuration are also shown [8]. Refer to the text for the correct interpretation of these plots.

As shown in Fig. 4 , the angular resolution of the detector does not suffer too much from the more frequent fluctuations present in the proton-induced showers. For iron induced showers we can see the overall tendency is the same, and the differences in angular resolution are negligible for practical purposes. In these figures we quote the previously published results for the HTVconfiguration [8]. The difference in results must be regarded with caution. Although we can see an improvement in the angular resolution for small zenith angles, we have a dramatic drop of successful angular reconstruction (around $50 \%$ for $\Theta=30^{\circ}$ ). The reason for this relies not so much in the change of detector but in the change of reconstruction techniques. The techniques used for the present work apply a more stringent "quality cut" on the events, than the techniques used in [8]. Therefore, at a first try, triggering events are discarded as non-re-constructable. Nevertheless in a real experiment scenario the coarser angular reconstruction would be applied in all those triggering events which could not be reconstructed on the first try. 

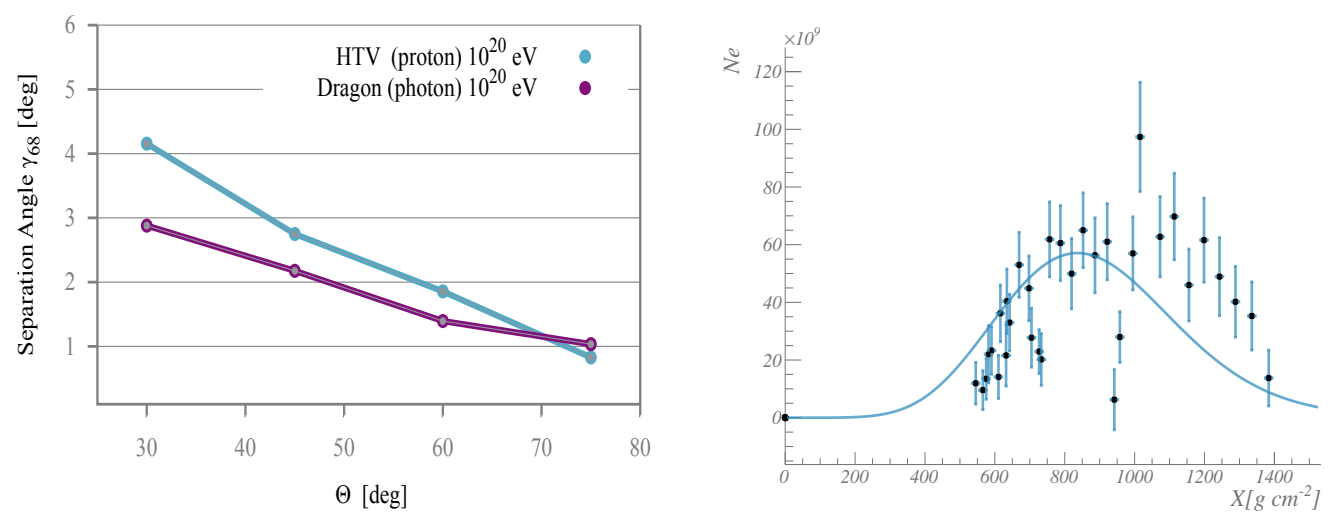

Figure 5: Left: The angular resolution as a function of the zenith angle of the Dragon-configuration of the JEM-EUSO mission for photon primaries with an energy of $10^{20} \mathrm{eV}$. Previous results with protons in the HTV-configuration are also shown (red line) [8]. Right An example of the reconstruction of the longitudinal profile of a proton-initiated EAS with an energy of $10^{20} \mathrm{eV}$ and $\Theta=60^{\circ}$. The points represent the reconstructed longitudinal profile with the associated uncertainty. The solid line is the originally simulated EAS.

\subsection{Angular reconstruction of photon primaries}

In the case of photons we suffer from a low triggering ratio for the showers that exhibit a strong Landau-Pomeranchuk-Migdal effect [3][4]. Showers with a strong LPM appear less bright, as a consequence of their extended longitudinal profile. The latter works as a selection filter only allowing the brightest photon showers to trigger the detector and are therefore available for reconstruction. Again this is mostly relevant at the lower zenith angles, whereas for higher zenith angles the impact is less dramatic. The angular resolution calculated using our photon sample is shown at the left side of Fig. 5

\section{Longitudinal profile reconstruction}

The longitudinal profile reconstruction available inside ESAF has only been optimized for parametrized proton showers [17] [13]. Nevertheless, we conducted a first blind test on our set of hybrid-simulated EAS applying the reconstruction module "as it is", but using more stringent quality cuts than what was previously used in [13]. We used results with Degrees of Freedom (DOF) bigger than 5; and $\chi^{2}<2$. An example of the reconstructed points of the shower compared to original shower can be seen in the right side of Fig. 5. We define the error in the energy reconstruction $\frac{\Delta E}{E}$ as: $\frac{\Delta E}{E} \equiv \frac{E_{\text {Reco }}-E_{\text {Simu }}}{E_{\text {Simu }}}$ where $E_{\text {Simu }}$ and $E_{\text {Reco }}$ are the simulated and reconstructed energies respectively; and in a similar way we define $\Delta X_{\max } \equiv X_{\max }^{R e c o}-X_{\max }^{\operatorname{Simu}}$ for the error in the $X_{\max }$ of EAS.

We show the results $E_{\text {Simu }}=10^{20} \mathrm{eV}$ for all angles in Fig. 6. To the left we can see the distribution of the error in the energy estimation. The first thing we notice is a systematic overestimation of the energy for all primaries. We can be confident that this can be corrected once a fine tuning of the reconstruction process is performed. However even with this systematic, the error is well within the $30 \%$ error budget of the mission. On the right side of Fig. 6 we see the corresponding plots for 

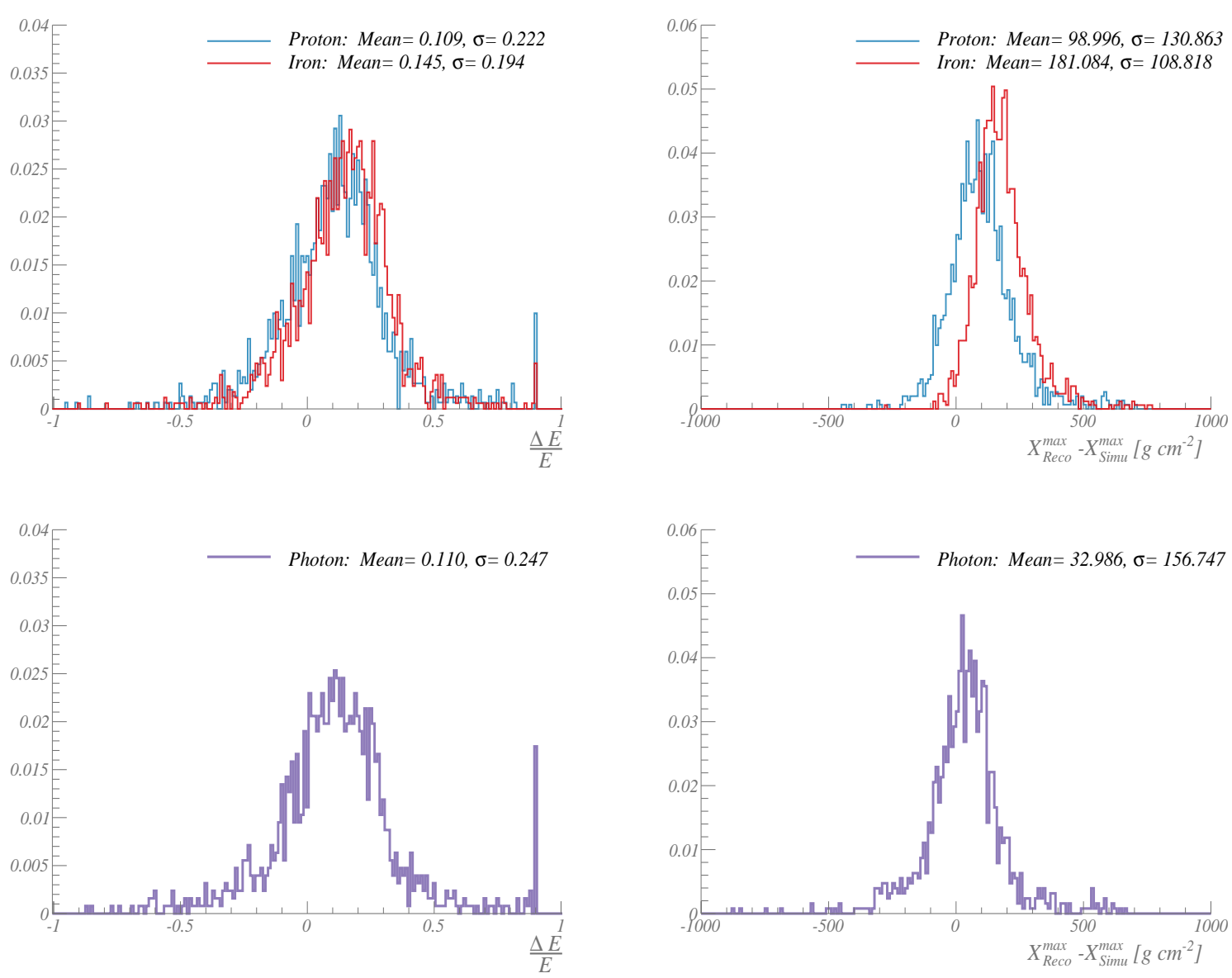

Figure 6: The energy-reconstruction error distribution (left) and the corresponding error distribution for $X_{\text {max }}$. (right). The uppper panel shows the results for protons and iron nuclei, and the lower panel for photons. In these plots the energy of the primaries was $10^{20} \mathrm{eV}$.

$\Delta X_{\max }$. Again we see a deviation from the ideal case $<\Delta X_{\max }>=0$ with a systematic overestimation of the $X_{\max }$ value. As before, we are confident a fine tuning can be performed to surmount this issue. More reassuring, is the fact that for both of the hadronic primaries the width of the $\Delta X_{\max }$ distribution is not too far off of the required performance of the mission (i.e. $\sigma_{X_{\max }} \sim 120 \mathrm{gcm}^{-2}$ ). Let us remember that these are the first tests of the tools developed in the context of the JEM-EUSO mission, outside of the specific conditions in which they were developed. For photon primaries we see a broader distribution of the errors, and the explanation is found by comparing the left and right sides of Fig. 3: the stronger LPM effect and the intrinsic higher fluctuations of the gamma shower complicate the reconstruction beyond what the ESAF module had been designed for.

\section{Conclusions}

We have presented a simulation-based analysis of the performance of the JEM-EUSO mission in its SpaceX Dragon configuration. For the first time, we explore the whole simulation and 
reconstruction chain using different types of primaries on the same footing. The first tests look promising and we have pointed out areas that still need improvement, for example the fine tuning of the longitudinal profile reconstruction to be more resilient to shower-to-shower fluctuations, and corrections for the systematics. The angular resolution seems to improve a little when compared with the HTV configuration, however this difference (if real) has to be thoroughly understood.

Acknowledgment: This work was partially supported by the ESA Topical Team Contract No. 4000103396 and by the Bundesministerium für Wirtschaft und Technologie through the Deutsches Zentrum für Luft- und Raumfahrt e.V. (DLR) under the grant number FKZ 50 QT 1101, by PAPIIT-UNAM/CONACyT, and by Basic Science Interdisciplinary Research Projects of RIKEN and JSPS KAKENHI Grant (22340063, 23340081, and 24244042), by the Italian Ministry of Foreign Affairs, General Direction for the Cultural Promotion and Cooperation, by the 'Helmholtz Alliance for Astroparticle Physics HAP' funded by the Initiative and Networking Fund of the Helmholtz Association, Germany, and by Slovak Academy of Sciences MVTS JEM-EUSO as well as VEGA grant agency project 2/0076/13.

\section{References}

[1] J.H. Adams Jr. et al. - JEM-EUSO Collaboration, Astroparticle Physics 44 (2013) 76

[2] A.D. Supanitsky, G. Medina-Tanco, A. Guzmán - JEM-EUSO Collaboration. Experimental Astronomy 10.1007/s10686-013-9353-2 (2013)

[3] L. Landau and I. Pomeranchuk Dokl. Akad. Nauk SSSR 92 (1953) 735-738

[4] A. Migdal Phys. Rev. 103 (1956)

[5] http://www.spacex.com/dragon

[6] J.H. Adams Jr. , R.M. Young, A. Olinto. - JEM-EUSO Collaboration, in proceedings of $33^{\text {rd }}$ International Cosmic Ray Conference (2013)

[7] http://global.jaxa.jp/projects/rockets/htv/

[8] S. Biktemerova, A. Guzmán, T. Mernik, - the JEM-EUSO Collaboration. Experimental Astronomy (2014) doi:10.1007/s10686-013-9371-0

[9] C. Berat, S. Bottai, D. De Marco, S. Moreggia, D. Naumov, M. Pallavicini, R. Pesce, A. Petrolini, A. Stutz, E. Taddei, A. Thea Astroparticle Physics Volume 33, Issue 4, (2010)

[10] T. Bergmann, R. Engel, D. Heck, N. N. Kalmykov, S. Ostapchenko, T. Pierog, T. Thouw, K. Werner Astropart. Phys. Vol. 26, pp 420-432, astro-ph/0606564 (2007).

[11] T. Pierog Journal of Physics: Conference Series 409 (2013) 012008

[12] P. Homola, et al. Computer Physics Communications Volume 173, Issues 1-2, Pages 71-90 (2005)

[13] F. Fenu, D. Naumov, - the JEM-EUSO Collaboration. Experimental Astronomy(s10686-2014-9427) (2014)

[14] William F. Hanlon,-The Telescope Array, Pierre Auger Collaborations.

[15] C. Bonifazi, for the Pierre Auger Collaboration, Nuclear Physics B (Proc. Suppl.) 190 (2009).

[16] T. Mernik PhD Dissertation, Universität Tübingen (2014)

[17] F. Fenu PhD Dissertation, Universität Tübingen (2013) 